Voix et Images

\title{
Entretien avec Jean-Marc Gouanvic
}

\section{Sophie Marcotte}

Volume 35, numéro 2 (104), hiver 2010

De l'anthologie

URI : https://id.erudit.org/iderudit/039162ar

DOI : https://doi.org/10.7202/039162ar

Aller au sommaire du numéro

Éditeur(s)

Université du Québec à Montréal

ISSN

0318-9201 (imprimé)

1705-933X (numérique)

Découvrir la revue

Citer ce document

Marcotte, S. (2010). Entretien avec Jean-Marc Gouanvic. Voix et Images, 35(2),

17-26. https://doi.org/10.7202/039162ar d'utilisation que vous pouvez consulter en ligne.

https://apropos.erudit.org/fr/usagers/politique-dutilisation/ 


\title{
ENTRETIEN AVEC JEAN-MARC GOUANVIC
}

\author{
$++$ \\ SOPHIE MARCOTTE \\ Université Concordia
}

Le genre de la science-fiction présente un intérêt particulier pour le chercheur en théorie et en histoire littéraires. Il n'y a pas beaucoup de littératures qui soient apparues à la période moderne et qui permettent d'analyser sur quelques décennies les déterminants contemporains de leur constitution. Ainsi, dans l'histoire du genre, est-il possible d'examiner le rôle et la place des romans, des nouvelles et des récits plus ou moins longs publiés dans des anthologies, des florilèges ou des chrestomathies. Le rôle joué par les anthologies de science-fiction québécoises est particulièrement pertinent dans le champ en formation, parce qu'il paraît généralisable aux autres champs littéraires. En effet, ce qui est constaté à propos de la sciencefiction est très probablement applicable aux autres genres littéraires (romanesques, poétiques, etc.) et les questions qui se posent au sujet des anthologies de sciencefiction risquent fort de se poser également pour les anthologies des autres genres. On peut, ce faisant, mieux saisir les manières d'être des agents dans les autres champs, mieux saisir les déterminants à l'œuvre dans ces derniers, à savoir les mécanismes fondés sur les luttes de pouvoir entre agents qui entendent promouvoir ce qu'il convient, à leur avis, d'admirer dans un genre littéraire. Or c'est bien l'un des rôles principaux de toute anthologie de tracer les voies d'avenir de la littérature à partir des productions d'un passé plus ou moins rapproché, et cela vaut pour la science-fiction comme pour toute autre production littéraire.

VoIX ET IMAGES Quel était le contexte lié à la publication de textes de science-fiction au Québec à la fin des années 1970?

JEAN-MARC GouAnvic Pour rendre compte de la publication des premiers textes de science-fiction issus du champ québécois, il faut remonter aux années 1977-1978, où la science-fiction en est au stade de l'émergence au Québec avec la création du fanzine Requiem par Norbert Spehner au cégep Édouard-Montpetit (Longueuil). Autour du fanzine s'est constitué un fandom qui se réunissait les premiers lundis du mois à l'hiver 1978. Je me souviens d'une réunion d'une trentaine de personnes, au début du mois d'avril 1978, à laquelle participaient Martin Fournier, Esther Rochon 
(auteure talentueuse de la science-fiction québécoise), Norbert Spehner, Gilbert Grand (alors journaliste au Devoir), Alain Lortie (alias Daniel Sernine), Bruce Robbins, Ann Charbonneau-Méthé (copropriétaire de L'Échange), Clodomir Sauvé (artiste d'avant-garde), Louis Picard, Michel Bélil (auteur de fantasy et futur membre de la rédaction d'imagine...). Même si ces réunions n'ont pas tenu très longtemps, elles sont le signe d'un engouement certain pour la science-fiction au Québec. L'idée de fonder une revue littéraire spécifiquement de science-fiction est née de rencontres avec Esther Rochon et Clodomir Sauvé. En 1979, ce fut imagine..., en format livre. Le titre est un emprunt direct à la célèbre chanson de John Lennon; le soustitre était «revue de science-fiction québécoise». Exactement à la même époque, Requiem se métamorphosait en Solaris, ce qui conférait à la science-fiction une place plus importante, car dans Requiem n'étaient publiés généralement que des textes brefs ou très brefs. Une rivalité extrêmement stimulante allait naître en 1979 de la coexistence de Solaris et d'imagine... dans le champ de la science-fiction. Puis, en 1980, Norbert Spehner fondait la première collection québécoise de science-fiction, "Chroniques du futur", aux Éditions Le Préambule (Longueuil) qui devaient y publier tout d'abord et simultanément deux recueils de nouvelles, le premier d'Élisabeth Vonarburg, L'œil de la nuit, et le deuxième de Jean-Pierre April, La machine à explorer la fiction.

VoIX ET IMAGES La création de ces revues a-t-elle servi de tremplin à la publication d'anthologies?

JEAN-MARC GouANvic C'est de l'expérience d'imagine... qu'est issu le désir de publier les anthologies, et en particulier la première, Les années-lumière, parue aux éditions VLB en 1983, qui opérait une sélection des récits de la revue imagine... parus entre 1979 et 1983.

volX ET IMAGES La revue imagine... et cette anthologie émanent donc du même projet?

JEAN-MARC Gouanvic Ces deux publications procèdent en effet d'une même conviction: tout d'abord, la conviction que la science-fiction est un genre spécifique et autonome, quoique assez proche de la fantasy (de la science fantasy), mais distinct du fantastique; ensuite, que la science-fiction est un genre véritablement littéraire (voir à ce propos la préface aux Années-lumière). La science-fiction est fondée sur l'évocation littéraire de l'altérité aussi bien historique que biologique et écologique, une évocation littéraire qui donne à la forme toute l'attention qui lui est due en littérature. Ce sont ces idées qui ont prévalu lors de la publication de ces textes, dans toute la mesure du possible. Je dis bien «dans toute la mesure du possible», car d'une part, il existe un courant de pensée au Québec, comme en France et aux ÉtatsUnis, qui tend à regrouper tous les genres antimimétiques sous une même bannière, généralement désignée par le terme «Fantastique», cela étant en partie dû à la tradition américaine, au sein de laquelle est né le courant de science-fiction au xxe siècle, qui s'attachait à faire coexister les trois fondateurs du genre, Jules Verne, H.G. Wells et Edgar Allan Poe (voir les premiers numéros d'Amazing Stories d'Hugo Gernsback et en particulier le $n^{\circ} 1 d^{\prime}$ 'avril 1926); d'autre part, parce que la science-fiction est un peu prisonnière de son héritage, de sa ghettoïsation issue du courant américain des années 1920. 
VoIX ET IMAGES Quel est l'objectif qui sous-tendait alors la publication d'anthologies de science-fiction d'un point de vue esthétique?

JEAN-MARC GOUANVIC L'objectif était de faire reconnaître la science-fiction comme un discours à part entière; il s'agissait de désenclaver le champ de la science-fiction en élargissant son public vers celui de la littérature, sans renoncer à la poétique que je crois détecter dans le genre. Toutes les initiatives d'édition que j'ai prises ont procédé de cette double exigence: publier des textes bel et bien littéraires sans renoncer à la poétique de la science-fiction.

voIX ET IMAGES Cette posture que vous avez adoptée semble vouloir faire sortir la science-fiction de la paralittérature, à laquelle elle est souvent associée d'emblée.

JEAN-MARC GOUANVIC Cette attitude militante, et parfois un peu agressive, venait de la constatation que, décidément, il n'était pas facile de lutter contre la place dominante des genres romanesques, dans le champ de la science-fiction aussi bien qu'en dehors. Le roman réaliste, genre mimétique dominant, occupait à peu près toute la place dans la réception critique, alors que le genre le moins mal aimé à cette époque était sans doute le genre fantastique. Ce n'est pas le lieu de détailler les causes d'un tel état de fait. Rappelons seulement qu'au XIX siècle, des écrivains importants tels que Charles Nodier, Guy de Maupassant et Théophile Gautier ont associé leur nom au fantastique. Il reste qu'il m'a toujours semblé un peu absurde que notre époque rejette les sciences et les techniques comme possibles thématiques en littérature, alors que ces sciences et ces techniques sont omniprésentes dans nos vies et qu'il ne serait pas inutile qu'on s'interroge sur leurs effets sociaux (ce que fait la sciencefiction).

voIX ET IMAGES Vous avez publié des anthologies et des recueils. Pouvez-vous nous rappeler la différence entre les deux d'un point de vue éditorial? Combien d'anthologies avez-vous préparées?

JEAN-MARC GouAnvic Au sens strict, j'ai publié deux anthologies: Les annéeslumière (VLB, 1983) et SF. Dix années de science-fiction québécoise (Éditions Logiques, 1988), qui sont des recueils de textes choisis, déjà publiés dans un autre contexte (imagine... dans le cas des Années-lumière et imagine... et Solaris dans le cas de SF. Dix années de science-fiction québécoise). Au sens large, une anthologie est également un recueil de textes qui n'ont pas déjà été publiés, mais qui n'en sont pas moins choisis; ce sont les quatre Espaces imaginaires (1983, 1984, 1985, 1986) publiés par les Publications Les Imaginoïdes (maison d'édition que j'avais créée pour publier la revue imagine...), les quatre recueils de nouvelles d'auteurs divers publiés dans la collection «Autres mers, autres mondes» des Éditions Logiques (de 1987 à 1991) et deux recueils d'auteurs précis (Annick Perrot-Bishop et Jean-François Somain). Les anthologies et les recueils de nouvelles allaient se heurter à une certaine réticence du public (qui venait s'ajouter aux autres déjà décrites auparavant) envers les textes brefs et les nouvelles. Pour contrer cette désaffection, j'allais proposer des recueils de courts romans, ce que les Américains nomment des novellas, en particulier dans la série des Espaces imaginaires, surtout le $n^{\circ} 3$ (1985) et le $n^{\circ} 4$ (1986).

voIX ET IMAGES A-t-il été difficile de trouver un éditeur pour ces anthologies de science-fiction? 
JEAN-MARC GOUANVIC En effet, il n'a pas été facile de trouver un éditeur pour Les années-lumière, vu le manque de légitimité de la science-fiction au Québec. VictorLévy Beaulieu, en tout premier lieu, et Jacques Lanctôt ont montré, en publiant cette anthologie, une ouverture d'esprit qui n'a pas manqué de conforter mes idées sur les possibilités offertes à la science-fiction québécoise. Financièrement, cependant, il n'était pas du tout dit que Les années-lumière allaient trouver un public assez nombreux qui permette de rentabiliser le livre (on sait combien l'impératif commercial impose à toute entreprise d'édition des normes strictes, trop de décisions hasardeuses risquant de mettre l'entreprise en péril), mais VLB acceptait de risquer une partie de sa légitimité d'éditeur dans la publication de cette anthologie explicitement proclamée de «science-fiction». Du point de vue financier, le calcul ne fut pas si mauvais, à en juger par le nombre d'exemplaires vendus (environ 750); cela n'était pas si mal pour une anthologie d'un genre «subalterne» présenté par un éditeur ayant pignon sur rue et publiant en général de la littérature du circuit lettré.

VoIX ET IMAGES Au plan symbolique, VLB a-t-il eu à regretter la publication de cette anthologie? A-t-il perdu quelques plumes dans l'affaire?

JEAN-MARC GOUANVIC Je n'ai pas d'indice de cela, mais j'en doute. Du point de vue de la science-fiction, les Années-lumière ont-elles eu quelque effet sur la reconnaissance du genre en dehors du champ restreint de la science-fiction? Pour répondre à cette question autrement qu'en se fondant sur des impressions, il aurait sans doute fallu que VLB poursuive la publication de science-fiction, romans, récits, anthologies, etc. Alors, on aurait pu voir quelle aurait été sa réception chez le public non assidu du genre. En tous cas, cette anthologie fut la seule que j'aie proposée à VLB. voIX ET IMAGES Comment Les années-lumière ont-elles été reçues par la critique? JEAN-MARC GOUANVIC Du point de vue de la science-fiction (agents du champ, auteurs et éditeurs), Les années-lumière furent une excellente opération, au moins jusqu'à la recension publiée dans Le Devoir, qui traita le livre avec beaucoup de désinvolture, le critique adoptant un point de vue très au-dessus de la mêlée. Cette douche froide me rappelait opportunément que les choses n'allaient pas changer aussi vite que je l'aurais souhaité...

Voix ET IMAGES Le premier tome des Espaces imaginaires a été publié en 1983, la même année que Les années-lumière. Pouvez-vous nous rappeler les circonstances qui ont présidé à la publication de cette série?

JEAN-MARC GOUANVIC Espaces imaginaires est une initiative dont j'ai eu entre les mains tous les éléments éditoriaux et techniques. Il m'avait semblé très pertinent, dès la fondation d'imagine..., d'ouvrir la science-fiction à l'ensemble de la francophonie et, pour cela, j'avais demandé à Stéphane Nicot, alors fanéditeur du fanzine français Espace libre (qui promettait beaucoup), de s'associer à la revue en dirigeant une chronique sur la science-fiction française. En 1983, nos relations avaient évolué vers l'édition de science-fiction, ce qui a pris forme avec la coédition d'Espaces imaginaires. En 1983 et 1984 devaient être publiés des auteurs français, belges et suisses, à côté des auteurs québécois, qui conservaient une sorte de primauté (par le nombre) dans les tomes de ces deux années.

VoIX ET IMAGES Espaces imaginaires a-t-il été bien reçu? 
JEAN-MARC GouANVIC À mon grand étonnement, Espaces imaginaires 1 a d'abord connu un franc succès auprès des institutions d'éducation, qui ont commandé en assez grand nombre des exemplaires par l'intermédiaire des agences d'abonnement (John Coutts, par exemple). L'anthologie n'était offerte que par envoi postal (la distribution est toujours le point névralgique des initiatives telles qu'Espaces imaginaires). L'argument décisif fut sans doute le prix d'achat, bas même pour l'époque (7,95 \$ l'exemplaire).

voix ET IMAGES Cette série a-t-elle obtenu la faveur du public et de la critique européens?

JEAN-MARC GOUANVIC La diffusion fut beaucoup plus difficile en Europe, malgré des recensions extrêmement élogieuses; le succès des Espaces imaginaires fut essentiellement canadien (pas seulement québécois). Cette anthologie a représenté pour moi l'occasion d'expérimenter une forme de récit de science-fiction particulièrement féconde en science-fiction américaine, la novella (court roman ou récit). En accord avec Stéphane Nicot, il fut décidé de publier des textes longs à partir de 1985: au lieu des dix auteurs habituels dans les anthologies et recueils d'alors, l'espace éditorial fut occupé par quatre auteurs en 1985 et par quatre autres en 1986. Cette forme de récit permet aux écrivains de déployer avec plus de liberté des univers de science-fiction fouillés, des intrigues travaillées répondant à l'exigence centrale du genre, l'évocation littéraire d'univers plausibles aussi fondamentalement autres qu'il est possible.

VoIX ET IMAGES Vous avez aussi participé à la fondation des Éditions Logiques, une maison d'édition essentiellement consacrée à la publication de textes de sciencefiction francophones.

JEAN-MARC GouANVIC En 1987, je crois, furent fondées les Éditions Logiques (par Louis-Philippe Hébert, président-directeur général, assisté de Roger Des Roches). «Participer à la fondation", c'est beaucoup dire. J'ai apporté un peu de mon savoirfaire de directeur littéraire en science-ficiton. Il avait paru naturel pour les Éditions Logiques de publier de la science-fiction, étant donnée leur "marque de fabrique», les sciences et les techniques. Louis-Philippe Hébert, auteur d'un roman remarqué (La manufacture de machines) et commanditaire pendant quelques années du Grand Prix de la science-fiction et du fantastique québécois, fut sensible à ma proposition de créer une collection de science-fiction, "Autres mers, autres mondes», aux éditions tout juste fondées. Jusqu'à 1991, onze livres de science-fiction ont été publiés, dont SF. Dix années de science-fiction québécoise en 1988, anthologie de dix nouvelles assez longues (mais non pas des novellas), et quatre recueils de nouvelles inédites comportant chacun un auteur français, belge ou suisse. SF. Dix années de science-fiction québécoise aurait peut-être pu connaître un meilleur sort auprès des lecteurs si l'anthologie avait été réalisée en format poche et à un coût moindre.

voix ET IMAGES Avez-vous fondé votre travail d'anthologiste ou d'éditeur de textes de fiction sur des modèles d'anthologies ou de recueils déjà publiés en Europe ou ailleurs dans la francophonie?

JEAN-MARC Gouanvic Mes idées de l'époque étaient en effet de suivre le modèle des anthologies de poche d'Hubert Juin, en particulier celle publiée en 1964 chez André Gérard dans la collection Marabout (Les vingt meilleurs récits de science-fiction, 
Verviers, Éditions Gérard, Bibliothèque «Marabout Géant»). Mais ces idées ne furent pas suivies, et les livres d'«Autres mers, autres mondes» ont été vendus relativement cher. La collection fut interrompue, faute d'avoir trouvé un public qui aurait accepté de payer le prix fort pour de la science-fiction. Soit dit en passant, «Autres mers, autres mondes» ne devait pas faire mieux que «Chroniques du futur» (Le Préambule), la collection de Norbert Spehner, qui avait aussi publié des anthologies (Aurores boréales).

voIX ET IMAGES L'horizon d'attente du lecteur d'anthologies est différent de celui du lecteur de romans. Comment expliquez-vous que la forme de l'anthologie suscite peu l'intérêt du lectorat populaire?

JEAN-MARC GouAnvic La publication d'anthologies et de recueils de nouvelles entraîne dans son sillage la question délicate de la publication de textes plus ou moins courts, en tout cas de textes qui ne sont pas des romans. Tous les éditeurs de la francophonie (le problème est le même en France) savent d'expérience que les anthologies et les recueils ont plus de difficultés à trouver un public que les romans. D'un autre côté, les nouvelles manifestent un dynamisme, une diversité culturels dans les champs littéraires. Ainsi, les années 1980 ont vu des «offensives» très énergiques allant dans le sens de la création de revues et de maisons d'édition visant à promouvoir la nouvelle dans le champ de la littérature réaliste; je songe à la revue XYZ (fondée en 1985) à Montréal, qui a pris pour sous-titre «la revue de la nouvelle", et à la maison d'édition L'instant même de Québec, qui s'attacha à publier des textes courts. Ces deux initiatives connurent une belle réussite et furent couronnées de prix littéraires. Mais, dans les années 1980, ces initiatives ne furent pas les seules. Je me souviens en particulier d'Arcade et de Stop.

VoIX ET IMAGES L'anthologie, par sa nature fragmentée, est-elle davantage destinée à être utilisée à des fins pédagogiques?

JEAN-MARC GouANVIC Une anthologie est par nature sélective. Elle représente en principe le meilleur de ce qui existe dans une littérature à un moment de son histoire. On peut donc très bien imaginer qu'une anthologie puisse servir à des fins pédagogiques. Je pense, comme je l'ai déjà mentionné, que les deux premiers tomes des Espaces imaginaires (1983 et 1984) ont été assez largement utilisés dans des institutions d'enseignement. Leur objectif n'était absolument pas pédagogique, mais ces institutions s'en sont emparées, me semble-t-il.

voIX ET IMAGES Voyez-vous l'anthologie comme une façon d'assurer une certaine longévité à des textes qui autrement disparaitraient de la mémoire collective? Dans le même ordre d'idées, un texte publié dans une anthologie de science-fiction telle que celles que vous avez préparées est-il susceptible de devenir un classique du genre?

JEAN-MARC GouAnvic Dans le champ de la science-fiction, la publication de textes courts était une tradition bien ancrée avec des magazines américains nombreux où ceux qui allaient devenir de grands auteurs (Bester, Dick, Bradbury, Leiber, Asimov, Padgett, van Vogt, Sturgeon...) ont presque tous fait leurs premières armes, les périodiques leur servant de bancs d'essai. Car il est indéniable que leurs nouvelles ont contribué à diffuser un genre et aussi une manière, et à faire admettre cette manière, mais il est rare qu'un auteur de science-fiction devienne un classique à la 
seule publication de nouvelles: ce sont les romans qui font les célébrités et qui assoient les légitimités.

VoIX ET IMAGES La science-fiction québécoise semble très influencée par la sciencefiction américaine. Cette influence est-elle passée par la traduction?

JEAN-MARC GOUANVIC La publication de la science-fiction, genre proclamé d'origine américaine, a dû, à un moment ou à un autre de son évolution dans les cultures non américaines, se définir par rapport aux grands classiques et donc prendre position sur la question de la traduction. Le Canada devait ajouter à cette problématique relevant de l'histoire littéraire d'un genre la problématique politique, avec la publication à partir de 1985 de l'anthologie Tesseracts (sous la direction de Judith Merril à Press Porcépic), qui fit une place à la science-fiction québécoise et construisit l'unité nationale "a mari usque ad mare» en science-fiction. Ce qu'il y a de remarquable dans cette construction, c'est qu'elle n'a jamais fait l'objet de discussion ni de débat, ce qui est un indice de l'absence de la question nationale en sciencefiction. En outre, il faut dire que, contrairement à la culture française qui construisit le champ de science-fiction sur la traduction des auteurs de science-fiction américains, l'émergence de la science-fiction québécoise se fit en dehors de la traduction des œuvres de science-fiction américaines. Cela est peut-être dû à ce que le fan de science-fiction au Québec lit l'anglais et a un accès direct aux œuvres américaines, en américain. C'est une situation qui contient des paradoxes, mais ces paradoxes ne sont pas nécessairement défavorables à la science-fiction québécoise francophone.

VoIX ET IMAGES Pour terminer...

JEAN-MARC GOUANVIC Quelle que soit la forme que les anthologies vont prendre dans les années à venir, c'est-à-dire quel que soit l'effet de la technologie sur elles, il me paraît douteux qu'elles soient amenées à jouer un rôle très différent de celui qu'on leur connaît aujourd'hui. Il est généralement admis que les littératures (et pas seulement les anthologies) dans les diverses langues s'étayent sur les cultures nationales (elles n'ont pas de réalité en dehors d'elles) et qu'elles contribuent à les former. On peut considérer qu'elles reposent sur des représentations identitaires et contreidentitaires qui construisent le continuum historique en mettant en discussion l'avenir social. Me permettez-vous de me lancer dans les conjectures les plus échevelées (très dans l'esprit de la science-fiction)? Ce type actuel de questions sur la littérature paraîtrait-il caduc, dans l'hypothèse d'une transformation radicale de la littérature par la technologie? Il faudrait sans doute que la littérature se métamorphose profondément en quelque chose d'autre pour que surgissent d'autres questions, qui seraient jugées plus centrales. Vous conviendrez qu'on est là dans les spéculations les plus hasardeuses. Si donc la littérature subit des transformations plus ou moins profondes, elles ne seront sans doute que de surface et les formes que prendraient les anthologies ne donneraient pas lieu à un type de questions différent de celui que nous énoncions plus haut. Mais s'il est une chose dont je suis sûr, c'est que l'avenir ne nous est pas accessible, c'est même ce qui le caractérise, et ces remarques sont faites de façon bien hypothétique. 


\section{A NTHOLOGIES ET RECUEILS DE TEXTES \\ DE SCIENCE-FICTION \\ préparés par Jean-Marc Gouanvic}

+ Les années-lumière. Dix nouvelles de science-fiction, Montréal, VLB, 1983, 240 p. Jean-Marc Gouanvic, «La science-fiction : une littérature à surveiller» Alexandre Amprimoz, «Le meurtre d'une idée» Jean-Pierre April, «Le fantôme du Forum » François Barcelo, «Écrivains XXIII » Michel Bélil, «L'angle parfait de Franco Bollo» André Carpentier, «La septième plaie du siècle» Agnès Guitard, «Les virus ambiance» Huguette Légaré, «Les trains-bulle de janvier» Michel Martin, «Vingt sommes» Jean Pettigrew, «Fragments d'une interférence : la maison close sur le nord» Esther Rochon, «Le labyrinthe» Jean-Marc Gouanvic, «Les auteurs»

+ Espaces imaginaires 1 (nouvelles), en collaboration avec Stéphane Nicot, Montréal, Les Imaginoïdes, 1983, 168 p.

Jean-Marc Gouanvic, «Présentation»

Agnès Guitard, «Coineraine»

Jacques Boireau, «Automne»

François Barcelo, «Les semeurs de robots»

Pierre Giuliani, «La frontière fictionnelle»

Esther Rochon, «Le traversier»

Daniel Walther, «Le dernier étage des ténèbres»

Jean-Pol Rocquet, "Gens de la Terre »

Gérard Gouesbet, «Une mort aux petits oignons»

Jean-François Somcynsky, «La triple flamme»

Stéphane Nicot et Jean-Marc Gouanvic, «Les auteurs»

+ Espaces imaginaires 2 (nouvelles), en collaboration avec Stéphane Nicot, Montréal, Les Imaginoïdes, 1984, 220 p.

Jean-Marc Gouanvic, «Présentation»

Daniel Paris, «La perle du pirate»

Daniel Sernine, «La tête de Walt Umfrey »

Christine Renard, «Le crocodile»

Jean Barbe, "Tous des apprentis »

Jean-Pol Rocquet, «Ruptures ou la mort ménin »

Pierre Sormany, «Le “Tyran" »

Michel Lamart, "Rêves de marionnettes»

Michel Bélil, «Rosemonde»

Jean-Pierre Planque, «L'archipel»

Marc Provencher, "Aplatir le temps»

Stéphane Nicot et Jean-Marc Gouanvic, «Les auteurs» 
+ Espaces imaginaires 3 (nouvelles), en collaboration avec Stéphane Nicot, Montréal,

Les Imaginoïdes, 1985, 166 p.

Jean-Marc Gouanvic, «Présentation»

François Rouiller, «Une enfance en jeu»

Colette Fayard, «Le Libérateur»

Alain Dartevelle, «Terrian Parade»

Esther Rochon, "Au fond des yeux"

Jean-Claude Dunyach, «Venez dans mon palais»

Jean-Pierre April, «Impressions de Thaï Deng»

Stéphane Nicot et Jean-Marc Gouanvic, «Les auteurs»

+ Espaces imaginaires 4 (nouvelles), en collaboration avec Stéphane Nicot, Montréal, Les Imaginoïdes, 1986, $174 \mathrm{p}$.

Jean-Marc Gouanvic, «Présentation»

Agnès Guitard, «Contre-courant»

Pierre Giuliani, «L'erreur de Balthazar»

Richard Canal, «Le dernier village»

Sabine Verreault, «Mané, Tékei, Pharès»

Stéphane Nicot et Jean-Marc Gouanvic, «Les auteurs»

+ Dérives 5. Anthologie permanente de la science-fiction francophone 1 (nouvelles), Montréal, Éditions Logiques, coll. "Autres mers, autres mondes», nº 1, 1988, 216 p.

Annick Perrot-Bishop, «Spirales de l'amour-mémoire»

Jean Pettigrew, «Biographie sommaire d'un émetteur-récepteur»

Jean-François Somcynsky, «Le procès Chronos»

Esther Rochon, «Devenir vivante»

Dominique Warfa, «Plongée profonde»

«Les auteurs»

+ SF. Dix années de science-fiction québécoise (nouvelles), Montréal, Éditions Logiques, coll. "Autres mers, autres mondes», n 3, 1988, 310 p.

Jean-Marc Gouanvic, "La science-fiction québécoise, un univers en expansion »

Jean-Pierre April, «Impressions de Thaï Deng»

Michel Bélil, «Travail de nuit: spectateur»

Alain Bergeron, «Les Crabes de Vénus regardent le ciel»

Bertrand Bergeron, «La vie de Faubourg»

Jean Dion, «La vie sur Mars»

Agnès Guitard, «Coineraine»

Annick Perrot-Bishop, "L'Ourlandine»

Esther Rochon, «Dans la forêt de vitrail»

Daniel Sernine, «Monsieur Olier devient ministre»

Jean-François Somcynsky, «2500»

«Les auteurs»

+ C.I.N.Q. Anthologie permanente de la science-fiction francophone 2 (nouvelles), Montréal, Éditions Logiques, coll. "Autres mers, autres mondes», n 4, 1989, 232 p. Jean-Marc Gouanvic, "Présentation. Cinq regards sur d'autres mondes» Jean Dion, «Au dieu marteau»

Colette Fayard, «Leçon de choses» 
Francine Pelletier, «Le tiers de l'avenir»

Michel Martin, «La tortue sur le trottoir»

Claude-Michel Prévost, «Pas de dum-dum pour Mister Klaus»

«Notices bio-bibliographiques»

+ Demain, l'avenir. Anthologie permanente de la science-fiction francophone 3 (nouvelles),

Montréal, Éditions Logiques, coll. "Autres mers, autres mondes», nº 9, 1990, 160 p.

Jean-Marc Gouanvic, «Demain, l'avenir»

Bertrand Bergeron, «La soustraction»

Victor Frigerio, «Des brebis et des hommes»

Marc Provencher, «Les hommes de la nuit»

Alain Bergeron, «Une analogie de la vie éternelle»

Georges Panchard, «Le dernier salon»

«Les auteurs»

+ SOL. Anthologie permanente de la science-fiction francophone 4 (nouvelles), Montréal, Éditions Logiques, coll. "Autres mers, autres mondes», nº 11, 1991, 246 p.

Jean-Marc Gouanvic, «Sol»

Guy Bouchard, «La chute»

Michel Lamart, «La part de sable»

Yves Meynard, "L'enfant des mondes assoupis»

Pierre Sormany, «Les univers parallèles d'Everett »

Alix Renaud, «Exanoïa»

«Les auteurs» 